\title{
Factores familiares frente al proceso académico de la niñez*
}

\author{
Family factors against the academic process of the child and the \\ girl* $^{*}$
}

\section{Fatores familiares contra o processo acadêmico da criança e a menina*}

\author{
Hugo Alexander Vega Riaño ** \\ Maribel Martínez Ochoa ***
}

\section{Universidad de Pamplona- Colombia}

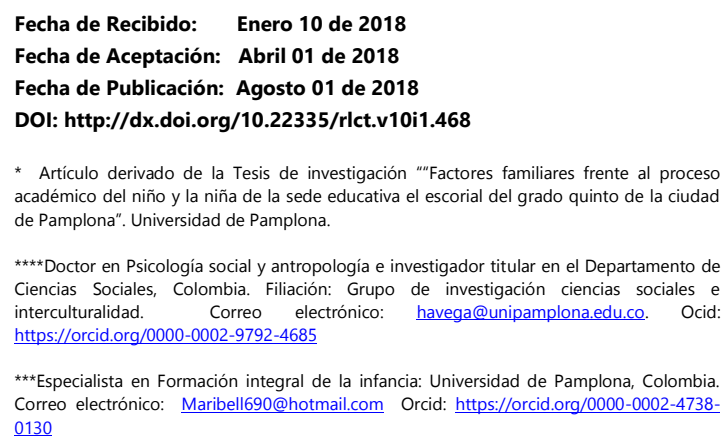

* Artículo derivado de la Tesis de investigación "'Factores familiares frente al proceso académico del niño y la niña de la sede educativa el escorial del grado quinto de la ciudad de Pamplona". Universidad de Pamplona.

****Doctor en Psicología social y antropología e investigador titular en el Departamento de Ciencias Sociales, Colombia. Filiación: Grupo de investigación ciencias sociales e interculturalidad. Correo electrónico: havega@unipamplona.edu.co. Ocid: https://orcid.org/0000-0002-9792-4685

***Especialista en Formación integral de la infancia: Universidad de Pamplona, Colombia. Correo electrónico: Maribell690@hotmail.com Orcid: https://orcid.org/0000-0002-4738$\underline{0130}$

\section{Resumen}

En el ambiente familiar se presentan un conjunto de relaciones que se establecen entre los miembros de la familia que comparten el mismo espacio (Bautista, F. 2006). Cada familia vive y participa en estas relaciones de una manera particular, de ahí que cada una desarrolle unas peculiaridades propias que le diferencian de otras familias; pero el ambiente familiar, sea como sea la familia, tiene unas funciones educativas $y$ afectivas muy importantes, ya que partimos de la base de que los padres tienen una gran influencia en el comportamiento de sus hijos e hijas y en su desempeño en el ambiente escolar.

Esta investigación analiza la familia como influye o no en el quehacer del educando o hasta qué punto esta puede determinar el alto o bajo nivel académico de los estudiantes de la sede educativa El Escorial, de tal manera que se pueda caracterizar, comparar y su vez determinar la importancia de la familia, sus relaciones, su aspecto socio-económico, su organización y la incidencia de estos factores con el fracaso o el triunfo escolar de un estudiante.

Palabras claves: Familia, composición familiar, derechos del niño, proceso educativo. 


\section{Abstract}

In the family atmosphere, a set of relationships established between family members who share the same space are presented. Each family lives and participate in these relationships in a particular way, hence each develop some peculiarities that differentiate it from other families; but the family atmosphere, whether as family, has very important educational and emotional functions, since we start from the premise that parents have a great influence on the behavior of their children and their performance in the school environment.

This research aims to analyze the family as influences or not in the work of the student or to what extent this can determine the high or low academic level of students of educational based $\mathrm{El}$ Escorial, so you can characterize, compare and turn determine the importance of the family, their relationships, their socio-economic aspect, their organization and the incidence of these factors in school success or failure of a student.

Keywords: Family, family composition, rights, educational process.

\section{Resumo}

No ambiente familiar, um conjunto de relações é estabelecido entre os membros da família que compartilham o mesmo espaço. Cada família vive e participa dessas relações de uma maneira particular, daí cada uma desenvolve suas próprias peculiaridades que a diferenciam de outras famílias; mas o ambiente familiar, seja qual for a família, tem funções educacionais e afectivas muito importantes, desde que começamos a partir da base de que os pais têm uma grande influência no comportamento de seus filhos e filhas e seu desempenho no ambiente escolar.

Esta pesquisa tem como objetivo analisar a família à medida que influencia ou não no trabalho do aluno ou em que medida pode determinar o alto ou baixo nível acadêmico dos alunos de el Escorial, de modo que possa ser caracterizado, comparado e ao mesmo tempo determinar a importância da família, suas relações, seu aspecto socioeconômico, sua organização e a incidência desses fatores com o fracasso ou o triunfo escolar de um aluno.

Palavras-chave: Família, composição familiar, direitos da criança, processo educacional.

\section{Metodología}

El trabajo se basa fundamentalmente en una investigación cualitativa de tipo descriptivo, partiendo en primer lugar el enfoque cualitativo se refiere según, Falla, Uva (2009), este enfoque no manipula las variables, ni efectúa la experimentación, este, además de hacer énfasis en aspectos subjetivos, del comportamiento del ser humano, su entorno, sus experiencias cotidianas, la interacción social y la relevancia de experiencias e interacciones; a medida que se presentan estas relaciones Taylor, S. y R.C. Bogdan (1989), nos indican que como se constituye las interpretaciones y los significados de la realidad del sujeto; esta investigación es de tipo descriptivo porque el propósito de la investigación es referir, situaciones, contextos y eventos, es decir detallar cómo se comportan, cómo se expresan por lo cual el estudio descriptivo busca puntualizar, las propiedades, las singularidades además de los rasgos delas personas, sus conglomerados, sus comunidades, la intensión es recolectar información sobre diversas variables o aspectos relacionados con el objeto a investigar (Hernández, S., Fernández, C.C., \& Baptista, L.P. (2006). 


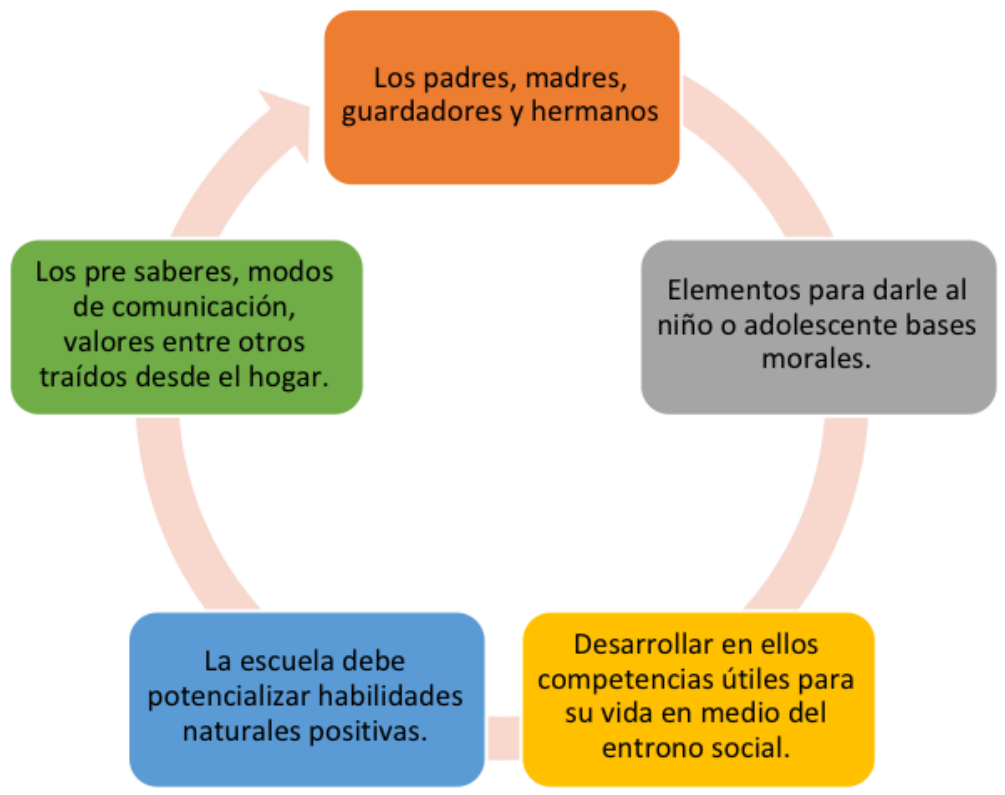

Figura 1. La educación y la familia. Fuente: Autores

Picado, K. (2007) nombra varios aspectos en pro de la educación de los niños y las niñas: Establecer una relación entre padres e hijos que acarree sentimientos de amor y respeto fraternal, de tal manera que, aunque se presenten situaciones negativas, siempre habrá relaciones donde predomine el afecto junto con el respeto por las reglas, límites establecidos dentro del núcleo familiar.

\section{El papel que ejerce la familia con relación al progreso del niño/a}

A través de esta se suplen necesidades básicas e indispensables para supervivencia de cada uno de sus miembros

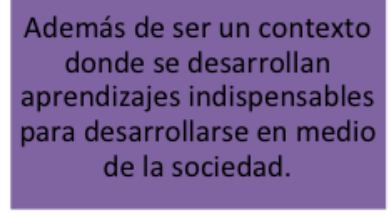

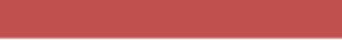

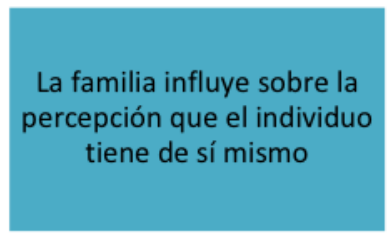

Teniendo en cuenta la información trasmitida por

los otros en este caso la ofrecida por los padres.

Figura 2. Familia y rendimiento académico. Fuente elaboración propia 
En relación con la vida escolar la familia ejerce autoridad sobre el comportamiento del aprendizaje y rendimiento del estudiante, ya que la actitud y conducta de los padres, sus creencias y expectativas son determinantes en relación con los talentos y resultados del niño y la niña, además ayuda al concepto positivo o negativo que tiene de él mismo, en relación al aspecto académico, así mismo del interés y la motivación por el cumplimiento de sus compromisos escolares (tareas), estos son aspectos determinantes en los resultados académicos obtenidos por el educando (Núñez, González\& González, 1994).

\section{Resultados}

Según lo dicho por Moreno, H., \& Chauta, R. C. (2011), los padres de familia consideran los procesos escolares como algo de vital importancia en la vida de sus hijos e hijas, tanto así que los motivan a que tengan un buen rendimiento académico porque piensan que la educación los puede ayudar a mejorar su calidad de vida, según Montes, I.C., \& Lerner, J. 2011), se pudo determinar que la mayor parte de los padres de familia no han podido culminar sus estudios de educación media, ya que por diversas circunstancias como embarazos a temprana edad y la necesidad de trabajar para sostener sus hogares fueron entorpeciendo y dejando a un lado sus aspiraciones educativas (Espina, 2004), la minoría que pudo terminar el bachillerato a través de la validación, cuando sus hijos ya tenían cierta edad, esta situación se presenta en familias con estructuras nucleares, donde el padre favorece estos procesos. De acuerdo a lo dicho por Moreno, D., Estévez, E., Murgui, S., \& Musitu, G. (2009), se determinó el interés de los padres para que sus hijos o hijas vean el provecho de la educación, ya que ellos expresaron el deseo de tener oportunidades de escolarización, porque comprenden que acceder a la educación puede mejorar las condiciones de vida de los miembros de la familia (Izar, J.M., Berenice, C. \& López, H. 2011).

Por lo anterior se manifiestan acciones por parte de los padres de familia en pro del quehacer escolar de los niños y las niñas similar a Burgos (2004), en el sentido de proveer de los materiales necesarios como: útiles, el provecho de las canastas escolares, el subsidio de uniformes, el cupo en el restaurante y la lonchera escolar, el mejor manejo de los recursos financieros obtenidos por medio de familias en acción, además de espacios propicios dentro del hogar donde puedan llevar a cabo sus actividades escolares, además del acompañamiento a las actividades que de desarrollan dentro del contexto escolar, la mayor participación es por parte de las madres.

\section{Discusión}

Con base a los resultados se puede concluir que el papel de la familia en relación con el rendimiento académico influye en cierta medida con la presencia de ambos padres según lo indicado por los siguientes autores Casals, A., \& Carrillo, C. 2015), y Bautista, F. (2006), Guevara, E.P., Jaramillo, R., \& Tovar, S. (2013), se deben proporcionar espacios que favorezca el desarrollo del niño y de la niña; espacios donde uno de los padres se dedique a actividades laborales y el otro se encargue de los aspectos relacionados con el hogar, donde se facilite acompañamiento real de las actividades extra clase, contrario alo propuesto por Benitez, O. S. (2016), y Benítez, S., Carillo De Albornoz, M., \& García Romero, J. C. (2015), en este caso de espacios adecuados que propendan por el desarrollo de las mismas; así mismo la participación activa de los padres en las actividades que se llevan a cabo en la escuela, por lo cual se deben favorecer los canales de comunicación, además de una efectiva relación entre la escuela y la familia, a fin de identificar y corregir posibles dificultades. 


\section{Conclusiones}

Se puede decir que el rendimiento académico de los niños y las niñas de la sede educativa el Escorial del grado quinto, en su mayoría está entre básico y bajo, un pequeño porcentaje está ubicado en los niveles alto y superior; se pudo evidenciar que las niñas son las que tienen los mejores resultados académicos, las áreas del conocimiento en las que se presenta bajo rendimiento académico son matemáticas y español, las que presentan los niveles altos son informática, educación estética y educación física, estas son las que más disfrutan los estudiantes y donde más se observa el interés; en el curso solo hay un caso de repitencia escolar, además de un grupo pequeño de estudiantes con extra edad (Espina, A. (2004).

Además es posible concluir que los factores que más inciden en el rendimiento escolar son en primer lugar, la composición familiar en este sentido, la familia y su estructura ejercen influencia en los resultados académicos de los estudiantes, ya que los estudiantes con niveles superior y alto en su gran mayoría pertenecen a un tipo de familia nuclear, donde es el padre quien trabaja para el sustento de su familia y la madre es quien se encarga la mayor parte del tiempo al cuidado de los hijos; en este aspecto también se pudo determinar que se presenta el caso de estudiantes que hacen parte de un núcleo familiar compuesto por ambos padres, donde los resultados están entre básico y bajo, evidenciando la falta de orientación de sus procesos escolares en el hogar (Guevara, E.P., Jaramillo, R., \& Tovar, S. 2013).

Se determinó que el desconocimiento real por parte de los padres del rendimiento académico del niño y la niña; en esta categoría de estructura - composición familiar se pudo observar estudiantes que pertenecen a familias monoparentales donde la mayor parte tienen niveles académicos básicos y bajos, en su rol como estudiantes demuestran inseguridad, apatía y desinterés, en algunos casos muestran sentimientos de tristeza por la ausencia del padre, además se presenta el otro extremo donde los estudiantes que viene de una composición familia monoparental, donde la madre se dedica a trabajar la mayor parte del tiempo, hay poco acompañamiento de actividades escolares, no cuenta con espacio propicio para estudiar y aun así tiene un rendimiento académico alto, a todo esto se puede decir que el sentido de superación, la responsabilidad y la autodisciplina son fundamentales en el querer ser el mejor y en el sentido de superación que se tiene.

\section{Referencias}

Bautista, F. (2006). Influencia del entorno familiar en la desmotivación escolar. Almería: Tágilis Ediciones.

Benitez, O. S. (2016). Single-sex education and right to education about the unconstitutionality on article 84.3 reform of the organic law of education. [Educación diferenciada por razón de sexo y derecho a la educación. Sobre la inconstitucionalidad de la reforma del artículo 84.3 de la ley orgánica de educación] Revista Espanola De Derecho Constitucional, 106, 451478. doi:10.18042/cepc/redc.106.12

Benítez, S., Carillo De Albornoz, M., \& García Romero, J. C. (2015). Respuesta endocrina a la aplicación de vibraciones de cuerpo completo en humanos. [Endocrine response to the application of Whole-Body Vibration in humans] Revista Andaluza De Medicina Del Deporte, 8(3), 109-114. doi:10.1016/j.ramd.2015.04.002

Burgos, J.M. (2004). Diagnóstico sobre la familia. (pp. 15-35). Madrid: Palabras S.A.

Casals, A., \& Carrillo, C. (2015). Best practices in music education: Construction of a classroom observation instrument. [Buenas prácticas en educación musical: Construcción de un 
instrumento para su observación] Opcion, 31(Special Issue 3), 384-406. Córdoba, L.G., Feu, S., García., Luengo, L.M., \& Vizuete. M (2011).

Espina, A. (2004). Familia, educación y diversidad cultural. (p.12). Universidad de Salamanca. Instituto de investigaciones Antropológicas de Castilla León: Aquilafuente.

Falla, Uva (2009). Reflexiones sobre la investigación social y el Trabajo Social. Tabula Rasa, mayojunio, no 10.

Guevara, E.P., Jaramillo, R., \& Tovar, S. (2013). Factores familiares y su relación con el rendimiento académico en estudiantes de psicología. Revista Virtual Universidad Católica del Norte, septiembre-diciembre.

Hernández, S., Fernández, C.C., \& Baptista, L.P. (2006). Metodología de la investigación. (4a . ed., p.102). México: Mc Graw Hill.

Izar, J.M., Berenice, C. \& López, H. (2011). Factores que afectan el desempeño académico de los estudiantes de nivel superior en Rioverde, San Luis Potosí, México. Revista de Investigación Educativa, 12.

Montes, I.C., \& Lerner, J. (2011). Rendimiento académico de los estudiantes: Perspectiva cuantitativa. Grupo de estudios en economía y empresa, Departamento de Desarrollo Estudiantil, Universidad EAFIT.

Moreno, D., Estévez, E., Murgui, S., \& Musitu, G. (2009). Relación entre el clima familiar y el clima escolar: el rol de la empatía, la actitud hacia la autoridad y la conducta violenta en la adolescencia. International Journal of Psychology and Psychological Therapy, 9(1), 123-13.

Moreno, H., \& Chauta, R. C. (2011). Funcionalidad familiar, conductas externalizadas y el rendimiento académico, en un grupo de adolescentes de un colegio distrital de la localidad de Usaquén de la ciudad de Bogotá. Revista Psychologia, 6 (1).

Núñez, J.C., González, J.A. \& González, P. (1994). Determinantes en el rendimiento académico. Variables contextuales y procesos cognitivomotivacionales. (305-308). España: Universidad de Oviedo.

Picado, K. (2007). El arte de aprender. Una guía formativa para el hogar y la escuela (pp. 24-85). San José, Costa Rica: Euned. 